\title{
Symmetry, dynamics and strings: a centennial issue in honor of Yoichiro Nambu
}

\author{
Bindu A. Bambah a \\ School of Physics, University of Hyderabad, C. R Rao Road, Hyderabad 500046, Telangana, India \\ Published online 12 February 2022 \\ (C) The Author(s), under exclusive licence to EDP Sciences, Springer-Verlag GmbH Germany, part of \\ Springer Nature 2022
}

\begin{abstract}
This short editorial provides an introduction to the special issue on the 100th birth anniversary of the legendary physicist Prof. Yoichiro Nambu. The special issue brings together various papers of some of the people whose work was inspired by Prof. Nambu's contributions to Physics.
\end{abstract}

With great pleasure and a sense of reverence, we bring forth this special issue of EPJ in honour of Prof. Y. Nambu. The title 'Symmetry, Dynamics and Strings' tries to encompass the broad areas of Prof. Nambu's contribution to Physics. In reality, his reach was so vast that no title would do justice to his immense contribution to Physics. January 18, 2021, was his $100{ }^{t h}$ birthday, and because of the pandemic, the celebrations were few and restricted to his students. However, the breadth and depth of Prof. Nambu's ideas warranted something on a much grander scale, considering all the physics his work has influenced. So, we decided to bring out a volume in which not only are his ideas showcased (no volume can do that), but people, who are "violets by a mossy stone", young physicists whose work is profoundly influenced by Prof. Nambu could pay homage. There have been special issues on Prof. Nambu from all over the world, but this one is special in a different way. It brings forth the influence he had on science in India. Prof. Nambu had three Indian students, Prof. Sumit R. Das (University of Kentucky and TIFR Mumbai), Dr Madhusree Mukerjee ( Scientific American, Germany) and Bindu A. Bambah (University of Hyderabad). This volume starts with a brief biography of Prof. Nambu by his biographer Dr Madhushree Mukerjee [1], which is informative and poignant at the same time[2-4]. It gives us insight into the inner workings of the brilliant mind of a person often called the 'Foreteller of Physics'. His ideas were way ahead of his time and continue to influence contemporary physicists. The article by Prof. Sumit Das [5] outlines the evolution of Nambu's breakthrough idea of string theory, which began as the physical description of the Veneziano model as vibrating strings for Hadrons $[6,7]$ and has now has taken a life of its own as one the most esoteric unification idea s

Guest editor: Bindu A. Bambah.

\footnotetext{
a e-mail: bbambah@gmail.com; bbambah@uohyd.ac.in (corresponding author)
}

in Physics [5,8]. Dr Soma Sanyal's article [9] on cosmic strings further illustrates the application of this pioneering idea $[10,11]$. His early work on the introduction of spontaneous symmetry breaking in particle physics, inspired by his interest in superconductivity, is reviewed by Prof. B. Ananthanarayan in his article [13] on 'Pions and Chiral Symmetry Breaking'. As Prof. Nambu himself says, "My discovery of spontaneous symmetry breaking (SSB) was an accidental event. But I may also say that I owe this to my education at Tokyo which had induced in me an interest in condensed matter physics, as well as to the proximity of Chicago to the birth place of the BCS theory" [12]. He goes on to say, "It took me about two years before I became convinced of the correctness of the BCS theory. In essence, I realised that a collective mode satisfied the WardTakahashi identity, thereby restored charge current conservation and gauge invariance. I also found that the SSB made the electromagnetic field acquire a mass and turn into a plasmon. It is a result of mixing the two zeromodes, the Coulomb field and the Nambu-Goldstone (NG) mode. Some condensed matter physicists gave a similar account, but I was quite satisfied that I was able to clarify the issue mathematically in terms of quantum field theory" [15]. In tune with this philosophy of analogy between condensed matter and particle physics systems, in this volume, Prof. Hiranmaya Mishra [14] looks at colour superconductivity in quark matter using the Nambu-Jona Lasinio model [16]. The article by Dr Harleen Dahiya [17] dwells on the quark structure of the proton in QCD, a theory inspired by Prof. Nambu's work. Many of Prof. Nambu's grand-students (students of students), Akshay Chatla, Abhishek Jha and Dr K.V.S Shiv Chaitanya, have contributed to this volume. The field of neutrino physics is one that interested Prof. Nambu a lot. This is due to the influence of Prof. Sakata in his life. In the immediate post-war period, he adhered to the Sakata-Taketani philosophy, a three-stage philosophy akin to dialectic materialism. In simple terms, 
this philosophy asserted that the evolution of particle physics goes through three stages: the search for regular patterns in the phenomena, the building of models to account for them, and the construction of abstract and precise mathematical laws $[12,18]$. He often mentioned these ideas. The neutrino mixing matrix is the Pontecorvo, Maki, Nakagawa and Sakata matrix. In his later years, he was interested in testing spontaneous breaking of Lorentz symmetry, and he felt neutrino systems would be ideal places to look for it [22]. Therefore we have included two papers on present-day neutrino physics, closely associated with experiments. One is by Dr Monojit Ghosh, and Prof. Rukmani Mohanta [19], and the other by Abhishek Jha and Akshay Chatla [20]. We could not get a contribution on Nambu Dynamics. However, Dr K.V.S Shiv Chaitanya's paper [21] illustrates how many different approaches in quantum dynamics such as random matrices and the Hamilton Jacobi method are connected. All in all, this issue contains topics as diverse as Prof. Nambu's ideas, and it is our centennial birthday tribute to him. We hope readers of this volume enjoy the articles and get some idea of the contributions of the impact of Prof. Nambu on the physics community, particularly the Indian physics community. This issue is only the tip of the iceberg. We hope it provides an impetus for the reader to derive inspiration from the work of one of the most unusual and creative minds of late 20thcentury Physics.

\section{References}

1. M. Mukerjee, An unassuming genius. Eur. Phys. J. Spec. Top. (2022). https://doi.org/10.1140/epjs/ s11734-021-00362-z

2. Madhusree Mukerjee, "Strings and Gluon:The Seer Saw Them All." Scientific American, 272(2): 3739 Feb (1995). https://www.scientificamerican.com/ article/strings-and-gluons-the-seer-saw-th/

3. M. Mukerjee, Profiles of Nambu, in Nambu: A foreteller of Modern Physics eds. T. Eguchi and M.Y Han, World Scientific Series in $20^{\text {th }}$ Century Physics, vol. 43, (2014), pp. xix-xxii

4. M. Mukerjee, Passing of a gentle genius, Huffington Post (2014). https://www.huffpost.com/entry/ the-passing-of-a-gentle-g_b_7827966

5. S. Das, QCD, strings and emergent space. Eur. Phys. J. Spec. Top. (2022). https://doi.org/10.1140/epjs/ s11734-021-00361-0

6. Y. Nambu, Quark model and the factorization of the Veneziano amplitude, in Lectures on the Copenhagen Summer Symposium, published in Broken symmetry: selected papers of Y. Nambu, vol. 13 (1970), pp. 258267

7. Y. Nambu, QCD and the string model. Phys. Lett. B 80(4-5), 372-376 (1979)

8. M.B. Green, J.H. Schwarz, Anomaly cancellations in supersymmetric $\mathrm{D}=10$ gauge theory and superstring theory. Phys. Lett. B 1984, 1007-1012 (1984)
9. S. Sanyal, Nambu Goto cosmic strings in the early universe. Eur. Phys. J. Spec. Top. (2022). https://doi.org/ 10.1140/epjs/s11734-021-00359-8

10. T.W.B. Kibble, J. Phys. A 9, 1387 (1976)

11. T. Vachaspati, A. Vilenkin, Phys. Rev. D 30, 2036 (1984)

12. Y. Nambu, Reminiscences of the youthful years of particle physics. Bull. Jpn. Phys. Soc. 57, 1 (2003) (Translated from Japanese in "Nambu: A foreteller of Modern Physics eds: T. Eguchi and M.Y Han", World Scientific Series in $20^{t h}$ Century Physics, Vol. 43,(2014), pp xiv -xvii)

13. B. Ananthanarayan, Pions: the original NambuGoldstone bosons. Eur. Phys. J. Spec. Top. (2022). https://doi.org/10.1140/epjs/s11734-022-00443-7

14. H. Misra, Colorsuperconductivity in magnetized quark matter: an NJL approach. Eur. Phys. J. Spec. Top. 12, 1 (2022). https://doi.org/10.1140/epjs/ s11734-022-00439-3

15. Y. Nambu, Quasiparticles and gauge invariance in the theory of superconductivity. Phys. Rev. 117, 6481 (1960)

16. Y. Nambu, G. Jona-Lasinio, Dynamical model of elementary particles based on an analogy with superconductivity. I. Phys. Rev. 122, 3481 (1961)

17. H. Dahiya, Flavor and spin structure of the proton. Eur. Phys. J. Spec. Top (2022). https://doi.org/10.1140/ epjs/s11734-022-00438-4

18. Y. Nambu, My memories of Sakata-Sensei. Progr. Theor. Phys. Suppl. 167, 175-178 (2007)

19. M. Ghosh, R. Mohanta, Updated sensitivity of DUNE in $3+1$ scenario with far and near detectors. Eur. Phys. J. Spec. Top. (2022). https://doi.org/10.1140/ epjs/s11734-021-00360-1

20. A. Jha, A. Chatla, Quantum studies of neutrinos on IBMQ processors. Eur. Phys. J. Spec. Top. (2022). https://doi.org/10.1140/epjs/s11734-021-00358-9

21. K. Haritha, K.V.S. Shiv Chaitanya, Random matrices and quantum Hamilton-Jacobi method. Eur. Phys. J. Spec. Top. (2022). https://doi.org/10.1140/epjs/ s11734-021-00363-y

22. P.G.O. Peter, Nambu at work. Progr. Theor. Exp. Phys. 2016(7), 07B106 (2016) 